\title{
Effect due to compositeness of nucleons in deep inelastic lepton nucleus scattering
}

\author{
Bo-Qiang Ma凡 \\ Institute of High Energy Physics, Academia Sinica, P.O.Box 918(4), \\ Beijing 100039, China \\ Institut für Theoretische Physik der Universität Frankfurt am Main, \\ Postfach 1119 32, D-6000 Frankfurt, Germany
}

\begin{abstract}
The off-shell behaviors of bound nucleons in deep inelastic lepton nucleus scattering are discussed in two scenarios with the basic constituents chosen to be baryon-mesons and quark-gluons respectively in light-cone formalism. It is found that when taking into account the effect due to internal quark structure of nucleons, the derived scaling variable for bound nucleons and the calculated nuclear structure functions are different from those in considering the baryon-mesons as the effective elementary constituents. This implies that the pure baryon-meson descriptions of nuclei give the inaccurate off-shell behavior of the bound nucleon structure function, thereby the quark-gluons seem to be the most appropriate degrees of freedom for nuclear descriptions. It is also shown that the EMC effect cannot be explained by nuclear binding effect from a sound theoretical basis.
\end{abstract}

\footnotetext{
* Fellow of Alexander von Humboldt Foundation.
} 


\section{Introduction}

What are the most appropriate degrees of freedom for nuclear descriptions? Can we develop a suitable effective quantum field theory of the baryonmeson that can accurately describe the detailed properties of the ordinary matter, or must we study the nuclear structure and dynamics in terms of the underlying quark-gluon component structure of hadrons? These questions are often asked by nuclear and particle physicists in recent years. One of the commonly accepted standpoints seems to be that nuclear physics on the microscopic level can be consistently described using nucleons, isobars and pions with some vector-meson contributions. In the low energy region where the constituent baryon-mesons can be considered as pointlike particles, this picture has been proved to be extremely successful. However, this picture may need improvement in the medium-and-high energy region where the compositeness of the hadrons should not be ignored further. In fact, it has been indicated by Brodsky several years ago [1] that the effective local Lagrangian field theory of the baryon-meson gives an inaccurate description of the actual dynamical and off-shell behaviors of hadronic amplitudes due to internal quark and gluon structure of the hadrons, hence it is not predictive and reliable. This viewpoint was stressed further recently by Jaroszewicz and Brodsky [2] from comparing the Z-diagrams in two pictures where the bound nucleons are treated as elementary Dirac particles and composite quark objects respectively. Jaffe also indicated [3] the limitations of the validity of effective field theory of mesons and baryons from comparing the calculated hadron properties from dispersion theory and those from quark model approaches.

This paper aims to emphasize Brodsky's viewpoint from the discussions

of the off-shell behaviors for bound nucleons in deep inelastic lepton nucleus scattering, i.e., the nuclear European Muon Collaboration (EMC) effect [4]. We will treat the scattering process in two scenarios with the basic constituents chosen to be baryon-mesons and quark-gluons respectively based on the light-cone quantum field theory [5]. The first scenario is to consider the nucleus as a bound state of baryon-mesons which are treated as 
effective elementary Dirac particles with the structure functions identified with those for free ones by a seemingly natural kinematic analogy. It is shown that the EMC effect could be phenomenally very well reproduced by using minimal multihadron Fock state (i.e., nucleons only) for certain $Q^{2}$ in taking into account the off-"'energy"-shellness of bound nucleons. However, the ambiguities in identifying the structure functions for bound nucleons seem to have not been avoided because there are ambiguities in evaluating the off-"'energy"-shell effect in this scenario. In order to avoid the off-"'energy"-shell ambiguities, we consider, in the second scenario, the nucleus as a bound state of baryon-mesons which are also composite states of quark-gluons. The scaling variable for bound hadrons is derived in a realistic way from the constraint of overall "'energy" conservation. It is found that this scaling variable for bound hadrons is different from that in the first scenario, hence we obtain different results for the nuclear structure function. The EMC effect cannot be explained by using minimal Fock state alone in the latter case if we do not introduce the "'intrinsic" distortions in the structure functions for bound nucleons caused by nuclear environment. Because the quark-gluon two-level convolution scenario is more reasonable in taking into account the correct dynamical and off-"'energy"-shell behaviors of the structure function for bound nucleons, the calculated nuclear structure function is more reliable than that in the pure baryon-meson scenario. Thereby this work indicates clearly that the compositeness of nucleons due to their internal quark-gluon structure should be taken into account in the study of high momentum transfer processes such as the nuclear EMC effect.

In Sect.2, the light-cone hadronic approach and the off-"'energy"-shell ambiguities are illustrated. The formulas of the quark-gluon two-level convolution scenario and some discussions are presented in Sect.3. Sect.4 gives the summary and conclusions. 


\section{The light-cone hadronic approach}

The EMC effect [4] is the fact that the measured ratios of the structure functions for bound nucleons to those for free ones are different from the earlier theoretical expectations [6] based on the impulse approximation in the rest frame of the nucleus. This effect was interpreted as a quark signature in nuclei when it was discovered[7]. Many models were proposed to explain the observed data by introducing non-nucleonic degrees of freedom such as nucleon swelling or overlap, multi-quark cluster, color conductivity, meson or isobar degrees of freedom, and quark exchange between nucleons etc. However, the situation has been more or less blurred since the appearance of a conventional nucleonic model [8] in which the EMC effect can be interpreted in terms of nucleonic degrees of freedom alone if the Fermi motion of nucleons and the nuclear binding effect (i.e., off-mass-shell effect) are taken into account. Because it was recognized that there are ambiguities in identifying the off-shell structure functions with the on-shell ones [9-10] and there is no a priori justification for ignoring final state interactions [11-12] in the conventional model, an alternative model in solving the above disadvantages simultaneously is obviously of importance for a further insight view of the EMC effect.

It was deemed, in several light-cone nucleonic models [12-14] which adopt the on-mass-shell kinematics for the bound nucleons, that the ambiguities in identifying the off-mass-shell structure function for bound nucleons with the on-mass-shell structure function for free ones are avoided. Furthermore, it has been indicated in ref.[12] that final state interactions can be ignored by using instant form dynamics in the infinite momentum frame, or using light-front form dynamics in an ordinary frame. Hence one may expect that the above two problems in conventional nucleonic approach can be avoided by using light-cone hadronic approaches in dealing with deep inelastic lepton nucleus scattering. In the following, we will exam whether this is the true case by discussing the off-shell behaviors in the pure baryon-meson scenario based on the light-cone quantum field theory of baryon-meson fields. 


\subsection{The pure baryon-meson scenario}

A relativistic composite model of nuclei in analogy with the relativistic composite model of hadrons developed by Brodsky et al. [5] has been proposed in ref.[12] by extending the light-cone quantum field theory to baryon-meson fields. This model has the advantage that the constituent baryon-mesons can be also visualized as composite systems of quark-gluons by employing the relativistic composite model of hadrons, with many of the nuclear properties in conventional nuclear physics still being detained. In this section, however, we will not introduce the internal quark structure of the baryon-mesons. We treat, instead, the baryon-mesons as the effective elementary constituents of the nucleus and use their off-shell structure functions to account for their compositeness.

It is adequate to use the minimal multihadron Fock state (i.e., nucleons only) in the discussions of the following sections because the main purpose of this paper is to investigate the role played by nucleonic degrees of freedom alone in the explanation of the EMC effect. By applying the impulse approximation, which has been justified in the light-cone approach[12], we can

illustrate the contribution to the hadronic tensor $W_{\mu \nu}^{A}$ for the target nucleus in Fig.1, where the dotted line means to sum over all possible final states. The kinematics for the particles are parametrized as

$$
\begin{gathered}
P_{\mu}=\left(P^{+}, P^{-}, \vec{P}_{\perp}\right)=\left(M_{A}, M_{A}, \overrightarrow{0}_{\perp}\right) ; \\
q_{\mu}=\left(q^{+}, q^{-}, \vec{q}_{\perp}\right)=\left(0,2 \nu, \vec{q}_{\perp}\right) ; \\
p_{\mu}=\left(p^{+}, p^{-}, \vec{p}_{\perp}\right)=\left(y P^{+},\left(M^{2}+\vec{p}_{\perp}^{2}\right) / y P^{+}, \vec{p}_{\perp}\right),
\end{gathered}
$$

provided with the defining equation

$$
q^{2}=-Q^{2} ; \quad P \cdot q=M_{A} \nu
$$

Using the calculation rules in ref.[12], we obtain

$$
W_{\mu \nu}^{A}=\sum_{B M} \int\left[d^{2} \vec{p}_{\perp} d p^{+} / 16 \pi^{3} p^{+}\right]\left[\rho_{B M}(\underline{p}) / y\right] W_{\mu \nu}^{N}\left(p, p^{\prime}\right),
$$

in which the function $\rho_{B M}(\underline{p})$ is the light-cone momentum (i.e., $\underline{p}=\left(p^{+}, \vec{p}_{\perp}\right)$ distribution of nucleons in the nuclear bound state, and $W_{\mu \nu}^{N}\left(p, p^{\prime}\right)$ is the 
hadronic tensor for the on-mass-shell struck nucleon with its kinematics before and after the scattering being $p_{\mu}$ and $p_{\mu}^{\prime}$ respectively, where $p_{\mu}^{\prime}$ subjects to the constraint of overall 4-momentum conservation between the virtual photon and the target nucleus. Then we have

$$
q^{\prime-}=p^{\prime-}-p^{-}=q^{-}+P_{A}^{-}-P_{C}^{-}-p^{-}=q^{-}-\left(\frac{\vec{p}_{\perp}^{2}+M_{C}^{2}}{M_{A}-p^{+}}+\frac{\vec{p}_{\perp}^{2}+M^{2}}{p^{+}}-M_{A}\right),
$$

where $M_{C}=\varepsilon+M_{A}-M$ is the mass of the residual nucleus and $\varepsilon=$ $M_{C}+M-M_{A}$ is the separation energy of a nucleon from the nucleus.

In ref.[12], the nuclear structure functions are obtained from the calculation of $L^{\mu \nu} W_{\mu \nu}^{A}$ in which the Z-graph contributions are neglected. In order to avoid the neglect of the Z-graph contributions, we calculate only the ++ component of $W_{\mu \nu}^{A}$ because there are no Z-graph contributions in it. Hence we obtain the convolution formula for the nuclear structure function

$$
F_{2}^{A}\left(\nu, q^{2}\right)=\sum_{B M} \int\left[\frac{d^{2} \vec{p}_{\perp} d p^{+}}{16 \pi^{3} p^{+}}\right] \frac{M_{A} \nu}{p \cdot q} y \rho_{B M}(\underline{p}) F_{2}^{N}\left(p, p^{\prime}, q\right),
$$

in which $F_{2}^{N}\left(p, p^{\prime}, q\right)$ is the structure function for the struck nucleon with $p_{\mu}$ and $p_{\mu}^{\prime}$ being the kinematics before and after the scattering, and $q_{\mu}$ being the 4 -momentum of the incident virtual photon. In a strict sense, the valve of

$F_{2}^{N}\left(p, p^{\prime}, q\right)$ is not the same as that for a free nucleon because the kinematics are different for the two cases. This arises the problem as to how to identify the off-shell structure functions for bound nucleons with those for free ones.

\subsection{The off-shell ambiguities}

\section{a) The on-shell case}

For a free nucleon at rest, we know its structure function can be expressed as

$$
F_{2}\left(p, p^{\prime}, q\right)=F_{2}\left(x, Q^{2}\right)
$$

with $x=Q^{2} / 2 M \nu$ and $Q^{2}=-q^{2}$. For a nucleon with velocity $\vec{v}$, its structure function can be written as

$$
F_{2}\left(p, p^{\prime}, q\right)=F_{2}\left(x^{\prime}, Q^{\prime 2}\right)
$$


with

$$
x^{\prime}=-q^{2} / 2 p \cdot q ; \quad Q^{\prime 2}=-q^{2} .
$$

Because the following relations hold for a free nucleon:

$$
p^{2}=M^{2} ; \quad q^{2}=-Q^{2} ; \quad q_{\mu}=p_{\mu}^{\prime}-p_{\mu},
$$

we may re-express $x^{\prime}, Q^{\prime 2}$ in different forms, such as by:

$$
x^{\prime}=Q^{\prime 2} / 2 M \nu^{\prime} ; \quad Q^{\prime 2}=\nu^{\prime 2}-\vec{q}^{\prime 2}
$$

with $\nu^{\prime}=\gamma(\nu-\vec{v} \cdot \vec{q}), \vec{q}^{\prime}=\gamma(\vec{q}-\nu \vec{v})$ and $\gamma=\left(1-\vec{v}^{2}\right)^{-1 / 2}$ as has been adopted by Noble [15], and by:

$$
x^{\prime}=-q^{2} / 2 p \cdot q^{\prime} ; \quad Q^{\prime 2}=-q^{\prime 2}
$$

with $q_{\mu}^{\prime}=p_{\mu}^{\prime}-p_{\mu}$ as will be adopted in this paper. In fact, there in an infinity $x^{\prime}$ and $Q^{\prime 2}$ with different expressions but same values for free nucleons.

\section{b) The off-mass-shell ambiguities}

The conventional nucleonic approach [8] is evaluated in the FerynmanDyson perturbation theory in the rest frame of the nucleus. Because 4momentum is conserved between the lepton and the struck nucleon, and the recoiling (A-1) nucleus is on-mass-shell, the struck nucleon must be off-massshell to ensure overall energy and momentum conservation. In this case, the relation $q_{\mu}^{\prime}=p_{\mu}^{\prime}-p_{\mu}=q_{\mu}$ holds but the on-mass-shell relation $p^{2}=M^{2}$ does not hold; i.e., the struck nucleon is off-mass-shell. From overall energy conservation we have $p_{\mu}=(M-\varepsilon, p)$.

In this case, eq.(2.10) equals to eq.(2.7) from $q_{\mu}^{\prime}=q_{\mu}$. However, eq.(2.9) does not equal to eq.(2.7) as was indicated in ref.[15]. In ref.[8], $x^{\prime}=-q^{2} / 2 p$. $q$ is adopted as the scaling variable for a bound nucleon and the EMCSLAC data are able to be reproduced by using nucleonic degrees of freedom alone in taking into account the consequences from the off-mass-shellness. However, it was argued by Noble in ref.[15] that $x^{\prime}=Q^{\prime 2} / 2 M \nu^{\prime}$ should be adopted as the scaling variable for a bound nucleon and that the nonnucleonic degrees of freedom are required for explaining the EMC effect 
by using this variable in the convolution formula. The above two methods both seem to be reasonable as illustrated by their users. This implies that the off-mass-shell ambiguities prevent reliable predictions of the off-massshell effect, and thereby the conventional nucleonic approach has difficulty in reliably evaluating the off-mass-shell effect.

\section{c) The off-"'energy"-shell ambiguities}

The light-cone approach in this paper is based on light-cone perturbation theory in which all particles are on-mass-shell, and $p^{+}$and $\vec{p}_{\perp}$ are conserved at every vertex with $p^{-}$not necessary conserved at every vertex. Thereby the relation $p^{2}=M^{2}$ holds but $q_{\mu}^{\prime}=q_{\mu}$ does not hold again. In this case the off-mass-shell ambiguities are avoided, as has been claimed in some previous literature. However, the off-shell ambiguities still exist because the off-"'energy"-shell ambiguities replace the off-mass-shell ambiguities now. From the calculation we know that the uses of $x^{\prime}=-q^{\prime 2} / 2 p \cdot q^{\prime}$ or $x^{\prime}=-q^{2} / 2 p \cdot q$ as the scaling variables for bound nucleons will give different results, as can be seen from Fig.2. The scaling variable $x^{\prime}=-q^{2} / 2 p \cdot q$ has been adopted in several light-cone hadronic approaches $[14,16]$ recently and it was found that the EMC effect cannot be reproduced by the nucleonic degrees of freedom alone. However, it was argued in ref.[17] that the use of $x^{\prime}=-q^{\prime 2} / 2 p \cdot q^{\prime}$ seems to be more natural than the use of $x^{\prime}=-q^{2} / 2 p \cdot q$ because the off-"'energy"-shell effect seems to have been considered. Thereby one may conclude from Fig.2 that the large part of the EMC effect could be explained by contributions from the nuclear Fermi motion effect and the nuclear binding (e.g., the off-"'energy"-shell effect) in terms of nucleonic degrees of freedom alone in the light-cone hadronic approach, as was done in the conventional nucleonic approach. Since the off-shell ambiguities have not been avoided in the light-cone hadronic approach, its predictions of the off-" 'energy"-shell effect are also unreliable as in the case of the conventional nucleonic approach. 


\section{The relativistic two-level convolution model}

The discussions in the precede section show that the off-shell ambiguities in identifying the scaling variable for a bound nucleon seem to have not been avoided in the light-cone hadronic approach. This can explain why there are prescription dependence in the calculated off-shell effect in nucleonic models, as was pointed out by Kisslinger and Johnson recently[16]. We know that the scaling variable for a free nucleon can be derived from the quark-parton model of hadrons $[18,5,19]$. Hence we may expect that the scaling variable for a bound nucleon can be also derived from the relativistic composite model of nuclei if the quark-gluon component structure of the hadrons are introduced. This inspired the author to have developed a relativistic twolevel convolution model for nuclear structure functions [20] with the scaling variable for a bound nucleon derived in a realistic way other than from assumptions.

\subsection{The quark-gluon two-level convolution scenario}

The scaling variable for a free nucleon has been derived in ref.[19] in the relativistic composite model of hadrons with the off-"'energy"-shell effect (i.e., the overall energy conservation effect called in ref.[19]) of the struck quark also considered. The calculation gave a good precocious scaling variable $x_{p}$, which reduces to the Weizmann variable, the Bloom-Gilman variable and the Bjorken variable at some approximations respectively. We now apply the technique in ref.[19] to derive the scaling variable for a bound hadron.

We introduce the quark-gluon component structure of the hadrons into the relativistic composite model of nuclei. In this scenario the target nucleus is considered as a composite system of hadrons which are also composite systems of quark-gluons. In neglecting quark interference corresponding to the quark exchange contributions, we can illustrate the contributions to the

hadronic tensor $W_{\mu \nu}^{A}$ for the target nucleus in Fig.3, where the reference frame is the same one in the first scenario and the kinematics for the quarks 
are parametrized as

$$
k_{\mu}=\left(k^{+}, k^{-}, \vec{k}_{\perp}\right)=\left(x p^{+},\left(m^{2}+\vec{k}_{\perp}^{2}\right) / x p^{+}, \vec{k}_{\perp}\right)
$$

Similar to the first scenario, we obtain

$$
W_{\mu \nu}^{A}=\sum_{B M} \int\left[\frac{d^{2} \vec{p}_{\perp} d p^{+}}{16 \pi^{3} p^{+}}\right] \frac{\rho_{B M}(\underline{p})}{y} \sum_{q} \int\left[\frac{d^{2} \vec{k}_{\perp} d k^{+}}{16 \pi^{3} k^{+}}\right] \frac{\rho_{B M}(\underline{k})}{x} w_{\mu \nu}\left(k, k^{\prime}\right)
$$

in which $\rho_{B M}(\underline{p})$ is the same one used in the first scenario, and

$$
\begin{gathered}
\rho_{q}(\underline{k})=\int \prod_{i=2}^{n}\left[\frac{d^{2} \vec{k}_{i \perp} d k_{i}^{+}}{16 \pi^{3} k_{i}^{+}}\right] 16 \pi^{3} \delta^{2}\left(\vec{p}_{\perp}-\vec{k}_{\perp}-\sum_{i=2}^{n} \vec{k}_{i \perp}\right) \\
\delta\left(1-x-\sum_{i=2}^{n} x_{i}\right)\left|\psi_{q}\left(\underline{p} ; \underline{k}, \underline{k}_{2}, \cdots \underline{k}_{n}\right)\right|^{2} ; \\
w_{\mu \nu}\left(k, k^{\prime}\right)=2 Q_{q}^{2}\left[k_{\mu} k_{\nu}^{\prime}+k_{\nu} k_{\mu}^{\prime}-g_{\mu \nu}\left(k \cdot k^{\prime}-m^{2}\right)\right] \\
\delta\left(k^{\prime-}+\sum_{j=2}^{m} k_{j}^{-}+\sum_{i=2}^{l} p_{i}^{-}-P^{-}-q^{-}\right) / k^{\prime+}
\end{gathered}
$$

The $x, y$ in the denominator of (3.2) are essential corresponding to the flux factors in ref.[21-23] which guarantee baryon number conservation, $\rho_{q}(\underline{k})$ is the quark momentum distribution in the struck baryon or meson, and $w_{\mu \nu}\left(k, k^{\prime}\right)$ is the hadronic tensor of the on-mass-shell struck quark with its kinematics before and after the scattering being $k_{\mu}$ and $k_{\mu}^{\prime}$ respectively, supplying $k_{\mu}^{\prime}$ subjects to the constraint of overall 4-momentum conservation between the virtual photon and the target nucleus[19]:

$$
\begin{gathered}
\vec{k}_{\perp}^{\prime}=\vec{q}_{\perp}+\vec{k}_{\perp} ; \\
k^{\prime+}=q^{+}+k^{+} ; \\
k^{\prime-}+\sum_{j=2}^{m} k_{j}^{-}+\sum_{i=2}^{l} p_{i}^{-}=P^{-}+q^{-} .
\end{gathered}
$$

To obtain $F_{2}^{A}=\nu W_{2}^{A}$, we calculate only the ++ component of $W_{\mu \nu}^{A}$ since the instantaneous fermion lines do not contribute to it as have been indicated by Brodsky et al.[5]. Using the relation

$$
F_{2}^{T}=\left(p_{T} \cdot q / 2 p_{T}^{+} p_{T}^{+}\right) W_{++}^{T}, \quad T=A, N,
$$

we obtain the two level convolution formula

$$
F_{2}^{A}\left(x, Q^{2}\right)=\sum_{B M} \int\left[\frac{d^{2} \vec{p}_{\perp} d p^{+}}{16 \pi^{3} p^{+}}\right] \frac{M_{A} \nu}{p \cdot q} y \rho_{B M}(\underline{p}) F_{2}^{N}\left(x_{B}, Q^{2}\right),
$$


where

$$
F_{2}^{N}\left(x_{B}, Q^{2}\right)=\sum_{q} \int d x \delta\left(x-x_{B}\right) x Q_{q}^{2} f_{q}\left(x, Q^{2}\right) \frac{q^{-}}{q^{-}+k^{-}}
$$

in which $f_{q}(x)$ is the quark distribution and $x_{B}$ is the scaling variable for the bound hadron. The factor $q^{-} /\left(q^{-}+k^{-}\right)$equals to 1 in the Bjorken limit. $x_{B}$ is obtained from the overall "'energy" conservation condition[19]

$$
\frac{\left[m^{2}+\left(\vec{k}_{\perp}+\vec{q}_{\perp}\right)^{2}\right]}{\left(k^{+}+q^{+}\right)}+\sum_{j=2}^{m} k_{j}^{-}+\sum_{i=2}^{l} p_{i}^{-}=\frac{\left(M_{A}^{2}+\vec{P}_{\perp}^{2}\right)}{P^{+}}+q^{-} .
$$

Replacing $\sum_{j=2}^{m} k_{j}^{-}$and $\sum_{i=2}^{l} p_{i}^{-}$by $p_{c}^{-}$and $P_{C}^{-}$, the minus component momentums of the residual hadron, nucleus respectively, we have

$$
x_{B}=(A-B) / 2 C,
$$

in which

$$
\begin{gathered}
A=C+m^{2}+\left(\vec{k}_{\perp}+\vec{q}_{\perp}\right)^{2}-m_{c}^{2}-\left(\vec{p}_{\perp}-\vec{k}_{\perp}\right)^{2} ; \\
B=\left\{A^{2}-4 C\left[m^{2}+\left(\vec{k}_{\perp}+\vec{q}_{\perp}\right)^{2}\right]\right\}^{1 / 2} \\
C=\left[M_{A}^{2}+2 M_{A} \nu-\left(\vec{p}_{\perp}^{2}+M_{C}^{2}\right) /(1-y)\right] / y .
\end{gathered}
$$

In the Bjorken limit $Q^{2} \rightarrow \infty, \nu \rightarrow \infty$ with $x=Q^{2} / 2 M \nu$ fixed, $x_{B}$ reduces to

$$
x_{B}=Q^{2} / 2 M_{A} \nu y .
$$

When comparing the above results with the calculation of the structure function for a free hadron in ref.[19], one sees that there are no ambiguities in identifying the structure functions for bound hadrons with those for free ones in assuming that there are no "'intrinsic" distortions in the quark distribution for bound hadrons caused by nuclear environment (e.g., nucleon swelling). However, the scaling variable for a bound hadron (i.e., $x_{B}$ in this paper) is different from that for a free hadron ( $x_{p}$ in ref.[19]) because the bound hadron is off-"'energy"-shell and subjects to the overall "'energy" conservation. In this sense, the binding effect (i.e., off-" 'energy"-shell effect) is also included in the two level convolution formula (3.7) from the constraint 
of overall "'energy" conservation. The contributions from nuclear binding are contained in $M_{C}$ in the scaling variable $x_{B}$, it gives $Q^{2}$ power-law type contributions and hence can be neglected if $Q^{2}$ and $\nu$ are sufficient large [19]. It becomes clear that the above result is different from that in the first scenario which gives a scaling variable $x^{\prime}=-q^{\prime 2} / 2 p \cdot q^{\prime}$ for a bound nucleon.

\subsection{The calculated results and discussions}

Fig.4 presents the calculated ratios $F_{2}^{A}\left(x, q^{2}\right) / F_{2}^{N}\left(x, q^{2}\right)$ in the quark-gluon two-level convolution scenario with the necessary inputs being the same as those used in ref.[12]. It is found that the results are different from those in the pure baryon-meson scenario. In the pure baryon-meson scenario, the large part of the EMC effect is able to be reproduced very well for $Q^{2}=5$ $(\mathrm{GeV} / \mathrm{c})^{2}$; whereas in the quark-gluon two-level convolution scenario the calculation cannot reproduce the data by using nucleonic degrees of freedom alone. The results in the quark-gluon two-level convolution scenario should be more reliable because the effect due to internal quark structure of nucleons is explicitly considered. Hence the results indicate that the EMC effect cannot be explained by nucleonic degrees of freedom alone if one properly considers the off-shell behaviors of nucleons due to their compositeness.

The difference between the baryon-meson and the quark-gluon scenarios can be seen from eq.(2.4) and eq.(3.7): The scaling variables for bound nucleons are different in the two scenarios though the kinematics for the struck nucleon are the same. In the pure baryon-meson scenario, the scaling variable for bound nucleons $\left(x^{\prime}=-q^{\prime 2} / 2 p \cdot q^{\prime}\right)$ is identified with that for free ones by a seemingly most natural kinematic analogy. Whereas in the quarkgluon two-level convolution scenario, the scaling variable for the bound nucleon (i.e., eq.(3.10)) is derived in a realistic way from the constraint of overall "'energy" conservation; i.e., in a way similar to that in which the Bjorken scaling variable was derived in the quark-parton model[18,5,19]. The difference hence indicates that the local Lagrangian field theory of the baryon-meson gives the inaccurate description of the actual dynamical and off-" 'energy"-shell behaviors of the structure function for bound nucleons 
due to their internal quark-gluon component structure.

We indicate here that the above conclusion does not rely on the specific form of the light-cone momentum distribution function $\rho_{B M}(\underline{p})$ used in this paper. The factor $\left(M_{A} \nu / p \cdot q\right) y$ in the two-level convolution formula eq.(3.7) equals to unity for large $Q^{2}$ and $\nu$. Glazek and Schaden have adopted a parton-like convolution formula, which is equivalent to eq.(3.7) for large $Q^{2}$ and $\nu$, to investigate the role of pionic degrees of freedom in the EMC-SLAC data [24]. From their analysis we know that the ratio $r$ will be equal to 1 around $x=0.6$, where the data show large deviation from 1 , if the nucleonic degrees of freedom alone are considered. This implies that non-nucleonic degrees of freedom are unambiguously required in the explanation of the EMC effect.

\section{Summary and conclusions}

The off-shell behaviors of bound nucleons in deep inelastic lepton nucleus scattering are discussed in two scenarios based on light-cone perturbation theory in this paper. It is shown that the off-" 'energy"-shell effect is not reliably handled in the pure baryon-meson scenario though the results may be good in describing the data (for discussions of some detailed features in the light-cone hadronic scenario, see ref.[17]). When taking into account the effect due to internal quark structure of hadrons, the EMC effect cannot be reproduced by using nucleonic degrees of freedom alone. This indicates that the nucleonic approaches have also the disadvantage that the effects due to compositeness of bound nucleons are unable to be reliably handled. Thereby after several years in elusiveness caused by the conventional nucleonic approach, the former conclusion [7] that the EMC unambiguously indicates the presence of non-nucleonic degrees of freedom seems to have been recovered. The inclusion of isobar and meson degrees of freedom (i.e., higher multihadron Fock state components), or the introduction of some other exotic effects such as the intrinsic distortions of the quark momentum distribution for hadrons caused by nuclear environment, quark exchange be- 
tween nucleons (or multiquark cluster), color conductivity, and hidden color component et al. are required in explaining the EMC effect.

One conclusion from this work is that it is probably inappropriate to treat the nucleus as a composite system of hadrons without considering their underlying quark-gluon structure in the medium and high energy physics regions. Hence much work should be done to analyze the effects due to compositeness of hadrons in various reactions and nuclear properties though they are not easy tasks. The questions asked in the beginning of this paper seem to have been answered by this work: The quark-gluons are essentially the most appropriate degrees of freedom for nuclear descriptions though the baryon-mesons are also the relevant degrees of freedom in nuclear physics.

\section{Acknowledgement}

The author would like to thank Prof.Tao Huang and Prof.Ji Sun for valuable discussions, and to acknowledge the encouraging discussions with Prof.M.B.Johnson. He is also grateful to Prof.W.Greiner for his hospitality and for the support from Institut für Theoretische Physik der Universität Frankfurt. 


\section{References}

1. S.J.Brodsky, Comments Nucl.Part.Phys.12(1984)213; in Short-Distance Phenomena in Nuclear Physica, eds. David H.Boal and Richard M.Woloshyn (Plenum, New York, 1983) p.141.

2. T.Jaroszewicz and S.J.Brodsky, Phys.Rev.C43(1991)1946.

3. R.L.Jaffe, Nucl.Phys.A522(1991)365c.

4. EMC, J.J.Aubert, et al., Phys.Lett.123B(1983)275;

A.Bodek, et al., Phys.Rev.Lett.50(1983)1431 and 51(1983)534.

For new EMC data, see, EMC, J.Ashman, et al., Phys.Lett.202B(1988)603.

5. S.J.Brodsky, in Lectures on Lepton Nucleon Scattering and Quantum Chromodynamics, eds. A.Jaffe and D.Ruelle (Birkhäuser, Boston, 1982) p.255;

S.J.Brodsky, T.Huang, and G.P.Lepage, in Particles and Fields, eds. A.Z.Capri and A.N.Kamal (Plenum, New York, 1983) p.143.

6. A.Bodek and J.L.Ritchie, Phys.Rev.D23(1981)1070; D24(1981)1400.

7. R.L.Jaffe, Phys.Rev.Lett.50(1983)228.

8. S.V.Akulinichev, et al., Phys.Rev.Lett.55(1985)2239; J.Phys.G11(1985) L245; Phys.Lett.158B(1985)485.

See also, e.g., B.L.Birbrair, et al., ibid.166B(1986)119; G.V.Dunne and A.W.Thomas, Nucl.Phys.A446(1985)437c; Phys.Rev.D33(1986)2061; Nucl.Phys.A455(1986)701.

9. M.B.Johnson and J.Speth, Nucl.Phys.A470(1987)488;

B.-Q.Ma and J.Sun, High Energ.Phys.Nucl.Phys.12(1988)337;

L.Heller and A.W.Thomas, Phys.Rev.C41(1990)2756.

10. A.W.Thomas, Prog.Part.Nucl.Phys.20(1987)21. 
11. R.L.Jaffe, Comments Nucl.Part.Phys.13(1984)39; in Relativistic Dynamics and Quark-Nuclear Physics, eds. M.B.Johnson and A.Picklesimer (Wiley-Interscience, New York, 1986) p.71.

12. B.-Q.Ma and J.Sun, J.Phys.G:Nucl.Part.Phys.16(1990)823.

13. E.L.Berger and F.Coester, in Workshop on Nuclear Chromodynamics: Quarks and Gluons in Particles and Nuclei, eds. S.J.Brodsky and E.J.Moniz, (World Scientific, Singapore, 1986) p.255;

See also, E.L.Berger, F.Coester, and R.B.Wiringa, Phys.Rev.D29(1984) 398; E.L.Berger and F.Coester, ibid.D32(1985)1071.

14. U.Oelfke, P.U.Sauer, and F.Coester, Nucl.Phys.A518(1990)593.

15. J.V.Nobel, in Symmetry Violations in Subatomic Physics, eds. by B.Castel and P.J.O'Donnell (World Scientific, Singapore, 1989) p.161.

16. L.S.Kisslinger and M.B.Johnson, Phys.Lett.B259(1991)416.

17. B.-Q.Ma, Mod.Phys.Lett.A6(1991)21.

18. S.D.Drell, D.J.Levy, and T.-M.Yan, Phys.Rev.187(1969)2159; ibid.D1 (1970)1035;

S.D.Drell and T.-M.Yan, Ann.Phys.(N.Y.)66(1971)578.

19. B.-Q. Ma, Phys.Lett.176B(1986)179;

B.-Q. Ma and J.Sun, Int.J.Mod.Phys.A6(1991)345.

20. B.-Q.Ma, Phys.Rev.C43(1991)2821.

21. L.L.Frankfurt and M.I.Strikman, Phys.Lett.183B(1987)254; Phys.Rep. 160(1988)235.

22. G.L.Li, K.F.Liu, and G.E.Brown, Phys.Lett.213B(1988)531.

23. C.Ciofi degli Atti and S.Liuti, Phys.Lett.225B(1989)215.

For similar discussions, see, S.Shlomo and G.M.Vagradov, Phys.Lett.232B (1989)19; S.V.Akulinichev and S.Shlomo, ibid.234B(1990)170. 
24. St.Glazek and M.Schaden, Z.Phys.A323(1986)451. 


\section{Figure Captions}

Fig.1. The contributions to the hadronic tensor $W_{\mu \nu}^{A}$ for the nucleus in the relativistic pure baryon-meson scenario.

Fig.2. The calculated ratio $F_{2}^{A}\left(x, Q^{2}\right) / F_{2}^{N}\left(x, Q^{2}\right)$ in the pure baryon-meson scenario in comparison with the data. The solid and dashed curves are the results with $q_{\mu}^{\prime} \neq q_{\mu}$ and $q_{\mu}^{\prime}=q_{\mu}$ respectively for $k_{F}=260 \mathrm{MeV}$, $Q^{2}=5(\mathrm{GeV} / \mathrm{c})^{2}$ and $\varepsilon=35 \mathrm{MeV}$. The points are the SLAC data for $\mathrm{A}=\mathrm{Fe}\left(Q^{2}=5(\mathrm{GeV} / \mathrm{c})^{2}, 8<\epsilon<24.5 \mathrm{GeV}\right)$ and the points are the new EMC data for $\mathrm{A}=\mathrm{Cu}\left(4.4(\mathrm{GeV} / \mathrm{c})^{2}<Q^{2}<40.4(\mathrm{GeV} / \mathrm{c})^{2}\right.$, $\epsilon=120-280 \mathrm{GeV}$ ) from ref.[4] respectively.

Fig.3. The contributions to the hadronic tensor $W_{\mu \nu}^{A}$ for the nucleus in the relativistic quark-gluon two-level convolution scenario.

Fig.4. The calculated ratio $F_{2}^{A}\left(x, Q^{2}\right) / F_{2}^{N}\left(x, Q^{2}\right)$ in the quark-gluon twolevel convolution scenario in comparison with the data. The solid curve are the results in the quark-gluon two-level convolution scenario for $k_{F}=260 \mathrm{MeV}, Q^{2}=5(\mathrm{GeV} / \mathrm{c})^{2}$ and $\varepsilon=35 \mathrm{MeV}$ with the dashed curve are the results in the pure baryon-meson scenario for comparison. 